\title{
Structure and optical properties of self-assembled multicomponent plasmonic nanogels
}

\author{
T. Cong, ${ }^{1}$ S. N. Wani, ${ }^{1}$ P. A. Paynter, ${ }^{1}$ and R. Sureshkumar ${ }^{1,2, a)}$ \\ ${ }^{1}$ Department of Biomedical and Chemical Engineering, Syracuse University, Syracuse, New York 13244, USA \\ ${ }^{2}$ Department of Physics, Syracuse University, Syracuse, New York 13244, USA
}

(Received 7 February 2011; accepted 1 July 2011; published online 29 July 2011)

\begin{abstract}
Multicomponent plasmonic nanogels (PNGs) capable of broadband absorption of light in the 400-700 nm wavelength range were synthesized by the self-assembly of metal nanoparticles with wormlike surfactant micelles. Small angle x-ray scattering and rheological experiments suggest that the nanoparticles bridge micelle fragments to aid the formation a stable gel phase with exceptional color uniformity. Their optical absorbance could be robustly tuned by changing the nanoparticle type (Au/Ag), size, shape, and/or concentration. The PNGs have relatively low viscosity and are thermoreversible. Potential applications to the manufacturing of coatings and interfaces for solar energy harvesting and reconfigurable optical devices can be envisioned. (C) 2011 American Institute of Physics. [doi:10.1063/1.3615785]
\end{abstract}

Improving the efficiency of thin-film photovoltaic (PV) devices using plasmonic interfaces is an area of active research. ${ }^{1}$ The incorporation of a light trapping layer made of noble metal nanoparticles (NPs) is a promising approach. ${ }^{2,3}$ Such nanostructured plasmonic interfaces have been fabricated by using lithography, ${ }^{4,5}$ vapor deposition, ${ }^{2,6}$ dewetting of thin metal films by $\mathrm{ns}^{7-11}$ and $\mathrm{fs}^{12}$ pulsed lasers and wet chemistry involving self-assembled monolayers. ${ }^{13}$ An economical scale up and adaptation of such processes for the fabrication of interfaces with multiple NP species/shapes/sizes in a controllable and repeatable fashion is not straightforward. ${ }^{14}$ In this letter, we show that an aqueous solution containing a network of wormlike surfactant micelles (WLMs) can be used as a template for producing stable multicomponent suspensions of $\mathrm{Au}$ and/or Ag NPs with desirable optical properties. Such suspensions, hereafter referred to as plasmonic nanogels (PNGs), exhibit a long shelf life ( $\sim$ weeks) and color uniformity as shown in Fig. 1. The shape, size, and concentration of the NPs can be varied to tune the optical properties of the PNGs. In this way, the PNGs can be designed to absorb radiation over a broad band of wavelengths. The multicomponent PNGs reported in this work have a relatively low viscosity and elastic modulus. So, they can be spin- or dip-coated onto substrates to produce plasmonic interfaces or be used in optofluidic devices. $^{15,16}$

Cetyltrimethylammonium bromide (CTAB) WLMs are elongated and semiflexible cylindrical aggregates formed by the self-assembly of amphiphilic surfactants in aqueous solutions. ${ }^{17,18}$ At sufficiently large concentrations, the micellar chains entangle to form a viscoelastic network with a linear dimension $\left(\xi_{\mathrm{M}}\right)$ on the order of $\left(k_{\mathrm{b}} T / G_{0}\right)^{1 / 3}$. Here, $G_{0}$ is the plateau storage modulus and $k_{\mathrm{b}}$ and $T$ represent the Boltzmann constant and absolute temperature, respectively. ${ }^{18}$ Fourier transform infrared spectroscopy and thermogravimetric analyses reported in the literature ${ }^{19}$ suggest that the headgroup of a CTAB molecule can bind to the surface of a

\footnotetext{
a) Author to whom correspondence should be addressed. Electronic mail: rsureshk@syr.edu.
}

NP. Further, the addition of NPs to an entangled WLM solution has been shown to influence the network structure through the formation of a double network of entanglements and NP-mediated junctions. ${ }^{20,21}$ We have characterized the structure of the PNGs with small-angle $\mathrm{x}$-ray scattering (SAXS) measurements. ${ }^{22}$ Fig. 2(a) shows the SAXS data for a $100 \mathrm{mM}$ CTAB micellar solution with and without the presence of monodisperse spherical $30 \mathrm{~nm}$ Au NPs (Nanopartz, Inc.). For the pure WLM solution, a Bragg diffraction peak was observed at a relatively large scattering vector $q \approx 0.053 \AA^{-1}$. This corresponded to a linear dimension $2 \pi / q$ $\approx 11.4 \mathrm{~nm}$ which is greater than the diameter of a single cylindrical micelle $(\approx 4.6 \mathrm{~nm})$. The diameter is known to be approximately twice the length of a CTAB molecule. ${ }^{23}$ Hence, the peak in the scattering intensity $I(q)$ signified the cross sectional dimension of the micellar aggregates. For $q \leq 0.01 \AA^{-1}$, the scattering intensity followed Porod's law, i.e., $I \sim q^{-4}$. This indicated a sharp interface between the micelles and the aqueous environment. ${ }^{24,25}$ For larger $q$ values, $I \sim q^{-1}$. This is consistent with a gel-like structure composed of cylindrical fibers. ${ }^{26}$ Upon the addition of Au NPs, an increase in $I(q)$ was observed for $q$ in the range of $0.01-0.04 \AA^{-1}$. The enhanced $I(q)$ could be fitted well to the form factor of a suspension with spheres of mean radius 16 $\mathrm{nm}( \pm 10 \%)$. The peaks became more pronounced as the NP

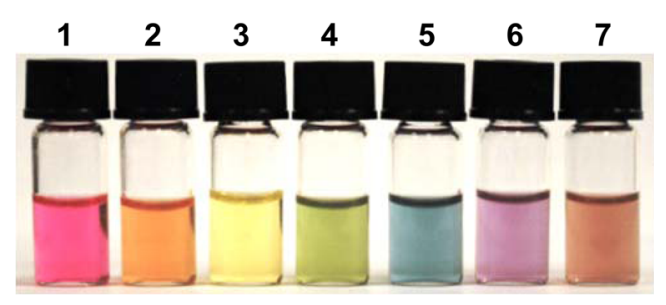

FIG. 1. (Color online) Multicomponent plasmonic nanogels with tunable optical properties. (1) $13.3 \mathrm{ppm} \mathrm{Au}(\mathrm{S}, d=30)$, (2) $8 \mathrm{ppm} \mathrm{Au}(\mathrm{S}, d=30)$ + $10 \mathrm{ppm} \operatorname{Ag}(\mathrm{S}, d=35)$, (3) $13.3 \mathrm{ppm} \operatorname{Ag}(\mathrm{S}, d=35)$, (4) $10 \mathrm{ppm} \operatorname{Ag}(\mathrm{S}$, $d=35)+10$ ppm Au (R, $\alpha=2.3)$, (5) 13.3 ppm Au (R, $\alpha=2.3)$, (6) 10 ppm $\mathrm{Au}(\mathrm{S}, d=30)+10 \mathrm{ppm} \mathrm{Au}(\mathrm{R}, \alpha=2.3)$, and (7) $6.7 \mathrm{ppm} \mathrm{Au}(\mathrm{S}, d=30)$ $+6.7 \mathrm{ppm} \operatorname{Ag}(\mathrm{S}, d=35)+5 \mathrm{ppm} \mathrm{Au}(\mathrm{R}, \alpha=2.3) . \mathrm{S}$ and $\mathrm{R}$ denote spherical and rod-like NPs, respectively. 

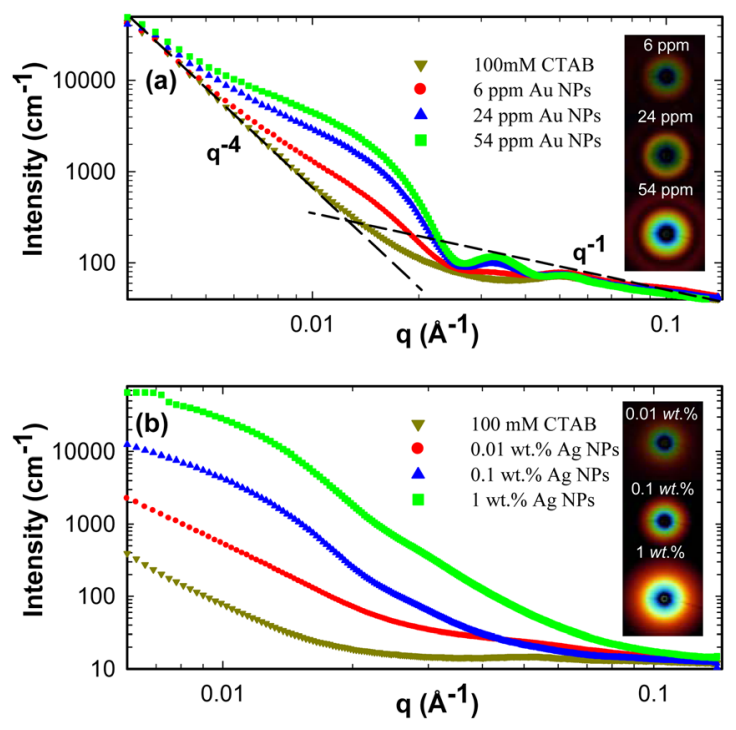

FIG. 2. (Color online) (a) SAXS data for plasmonic gels with $30 \mathrm{~nm} \mathrm{Au}$ NPs. (b) SAXS data for plasmonic gels with polydisperse Ag NPs.

concentration was increased. Hence, the increase in the scattering intensity was attributed to the presence of the dispersed NPs. ${ }^{22}$ A peak corresponding to the particles' mean nearest neighbor distance was observed at $q=0.0114 \AA^{-1}$. The corresponding distance, $55.4 \mathrm{~nm}$, is approximately twice the NP diameter. Hence, the NPs were assumed to be uniformly distributed without agglomeration. Further, for $q \geq 0.06 \AA^{-1}$, the addition of NPs did not significantly affect the scattering intensity. This indicated that the local micellar structure was preserved for length scales up to $\sim 10 \mathrm{~nm}$. A comparison of the SAXS data for $100 \mathrm{mM}$ CTAB samples with $0,0.01,0.1$, and 1 wt. \% polydisperse Ag NPs (Sigma-Aldrich, average diameter $d_{\text {avg }} \approx 38 \mathrm{~nm}$ ) is shown in Fig. 2(b). Local micellar structure was seen to be preserved in this case as well.

The structural information deciphered from SAXS data was complemented by linear and nonlinear rheological measurements on $100 \mathrm{mM} \mathrm{CTAB} / 200 \mathrm{mM} \mathrm{NaNO}_{3}$ samples with 0 , 0.01 , and 0.1 wt. $\%$ Ag NPs. ${ }^{22}$ A TA Instruments' AR-G2 rheometer was used. The storage and loss moduli $G^{\prime}$ and $G^{\prime \prime}$ were fitted to the Maxwell model to determine the structural parameters and the stress relaxation time $\lambda_{\mathrm{r}}$. As shown in Table I, the mesh size of the micellar network decreased from 76 to 58 $\mathrm{nm}$ as NP concentration was increased from 0 to $0.1 \mathrm{wt}$. $\%$. The increase in $G_{0}$ observed upon the addition of the NPs can be interpreted as a commensurate increase in the network density $\rho=G_{0} / k_{\mathrm{b}} T$ that is caused by the formation of NP-mediated network junctions. The observed three-fold increase in the zero shear viscosity, $\eta_{0}$, for the sample with 0.1 wt. $\% \mathrm{Ag}$ NPs is much greater than what the Einstein equation for the viscosity of dilute suspensions would predict. ${ }^{27}$ This is a further indication of the formation of micelle-NPs junctions.

The optical absorbance of the PNGs was measured using a UV-VIS spectrometer (Ocean Optics, Inc., USB-4000). PNGs containing spherical Ag, spherical $\mathrm{Au}$ and/or rod-like Au NPs (Nanopartz, $d_{\text {avg }} \approx 25 \mathrm{~nm}$, aspect ratio ranging from 1.4 to 3 ) were studied. ${ }^{22}$ The compositions of nine representative samples I-IX with different NP types (A-F) are listed in Table II. The absorbance increases linearly with concentration for relatively low NP concentrations. ${ }^{28}$ Hence, the addition of
TABLE I. Structural and rheological parameters of Ag-WLM PNGs. Linear rheological data were fitted to the Maxwell model. Nonlinear rheological data were fitted to Carreau model.

\begin{tabular}{lcccc}
\hline \multicolumn{1}{c}{ Property } & 0.00 wt. $\%$ & 0.01 wt. $\%$ & 0.05 wt. $\%$ & 0.10 wt. $\%$ \\
\hline$\eta_{0}(\mathrm{~Pa} \cdot \mathrm{s})$ & 1.32 & 1.36 & 1.70 & 3.47 \\
$\lambda_{\mathrm{r}}(\mathrm{s}$, nonlinear $)$ & 0.101 & 0.112 & 0.134 & 0.192 \\
$G_{0}(\mathrm{~Pa})$ & 9.34 & 13.23 & 17.25 & 21.43 \\
$\rho\left(\times 10^{21} \mathrm{~m}^{-3}\right)$ & 2.27 & 3.21 & 4.20 & 5.21 \\
$\xi_{\mathrm{M}}(\mathrm{nm})$ & 76 & 68 & 62 & 58 \\
$\lambda_{\mathrm{r}}(\mathrm{s}$, linear $)$ & 0.103 & 0.108 & 0.117 & 0.201 \\
\hline \hline
\end{tabular}

multiple species of NPs resulted in an absorbance spectrum, which was a linear superposition of the individual spectra.

Fig. 3(a) shows the extinction spectra of five two-component samples I-V. Samples I (spherical and small aspect ratio rod-like NPs), III, IV, and V (multicomponent spherical NPs) have only two peaks in their spectra. Each peak corresponds to the radial plasmon resonance of the individual constituent. The longitudinal plasmon resonance peak coincides with the radial peak for particles with a small aspect ratio. ${ }^{28}$ The superposition of the off-resonant tails of the individual spectra led to an absorption in the off-resonant regions that were in between the two resonant peaks. Longitudinal plasmon resonance in the large aspect ratio NPs (A and E) of sample II led to an additional characteristic peak. Samples VI-IX with three or more NP species were prepared to obtain a more uniform extinction spectrum [Fig. 3(b)]. Sample VI, which contained equal amounts of NPs A-E, showed three absorption peaks located at $\approx 410 \mathrm{~nm}, \approx 520 \mathrm{~nm}$, and $\approx 680 \mathrm{~nm}$. The first two peaks were due to the radial plasmon resonance of species $\mathrm{A}-\mathrm{C}$, the third peak was attributed to the longitudinal plasmon resonance of species D and E. The peaks corresponding to the radial plasmon resonance of species D and E were small and diffuse in comparison. Hence, they contributed by increasing the overall absorbance in the $400-600 \mathrm{~nm}$ range. The ability to tune the spectrum was explored further with the following guiding rules that were derived from our experiments and the literature: ${ }^{28,29}$ (1) Tuning the relative proportion of the NPs affected the uniformity of the spectra; e.g., samples VI and VII had the same total weight fraction, but an increase in the concentration of large aspect ratio rod-like Au NPs (E) in sample VII led to a more uniform spectrum. (2) An increase in the proportion of large diameter spherical NPs led to broader red-shifted peaks. So, the presence of NP species C in sample VIII led to the appearance of a broader red-shifted peak near $520 \mathrm{~nm}$. (3) The longitudinal plasmon resonance of NPs with a moderate aspect ratio lies in the red region. Rod NPs (D) with an aspect ratio of 2.3 gave rise to a peak that was near $620 \mathrm{~nm}$ in samples VI and IX. The presence of this peak gave rise to an improved uniformity of the spectrum.

In conclusion, we have shown that multicomponent plasmonic nanogels with a broadband absorption capability can be robustly synthesized by the self-assembly of metal NPs with wormlike micelles in an aqueous solution. While we focused on the visible range of the solar spectrum, it is plausible to access the UV and/or IR regions by a suitable selection of the constituent species such as different metals 
TABLE II. Composition (in weight fractions) of multiple species (A through F) plasmonic gels. The WLM concentration is $100 \mathrm{mM}$ for all samples. The diameter of spherical NPs (S) is denoted by $d$ while $d_{\text {rod }}$ and $\alpha$ represent the diameter and aspect ratio of rod-like NPs (R).

\begin{tabular}{|c|c|c|c|c|c|c|}
\hline Sample & $\begin{array}{c}\mathrm{A}(\mathrm{Ag}, \mathrm{S}) \\
d=35 \mathrm{~nm}(\mathrm{ppm})\end{array}$ & $\begin{array}{c}\mathrm{B}(\mathrm{Au}, \mathrm{S}) \\
d=30 \mathrm{~nm}(\mathrm{ppm})\end{array}$ & $\begin{array}{c}\mathrm{C}(\mathrm{Au}, \mathrm{S}) \\
d=90 \mathrm{~nm}(\mathrm{ppm})\end{array}$ & $\begin{array}{c}\mathrm{D}(\mathrm{Au}, \mathrm{R}) \\
d_{\mathrm{rod}}=25 \mathrm{~nm} \alpha=2.3(\mathrm{ppm})\end{array}$ & $\begin{array}{c}\mathrm{E}(\mathrm{Au}, \mathrm{R}) \\
d_{\mathrm{rod}}=25 \mathrm{~nm} \alpha=3.0(\mathrm{ppm})\end{array}$ & $\begin{array}{c}\mathrm{F}(\mathrm{Au}, \mathrm{R}) \\
d_{\mathrm{rod}}=25 \mathrm{~nm} \alpha=1.4(\mathrm{ppm})\end{array}$ \\
\hline I & 26.7 & - & - & - & - & 21.3 \\
\hline II & 26.7 & - & - & - & 21.3 & - \\
\hline III & 13.3 & - & 21.3 & - & - & - \\
\hline IV & 26.7 & 21.3 & - & - & - & - \\
\hline V & 13.3 & 21.3 & - & - & - & - \\
\hline VI & 6.7 & 6.7 & 6.7 & 6.7 & 6.7 & - \\
\hline VII & 3.3 & 6.7 & 6.7 & 3.3 & 13.3 & - \\
\hline VIII & 6.7 & - & 13.3 & - & 13.3 & - \\
\hline IX & 3.3 & 3.3 & 13.3 & 6.7 & 6.7 & - \\
\hline
\end{tabular}

and varying NP architectures. ${ }^{29}$ The self-assembly route described in this letter is a scalable room temperature process and does not rely on specific chemical interactions. Therefore, it is applicable to a variety of metals and shapes. Further, the gels have a long shelf life and are thermally stable up to $\approx 80^{\circ} \mathrm{C}$. The network melted at temperatures $>80^{\circ} \mathrm{C}$, resulting in a phase separated system consisting of a dense NP layer beneath a transparent surfactant solution. However, upon cooling to below $80^{\circ} \mathrm{C}$ followed by vortex mixing, the PNGs were reformed. Thus, the phase transition is thermoreversible. Since the PNGs possessed a relatively low viscosity $(\sim 1 \mathrm{~Pa} \cdot \mathrm{s})$ and low elasticity, they can be processed by wellestablished low cost techniques such as dip-/spin-coating onto or spraying over substrates to produce thin films and interfaces that are potentially suitable for applications rang-
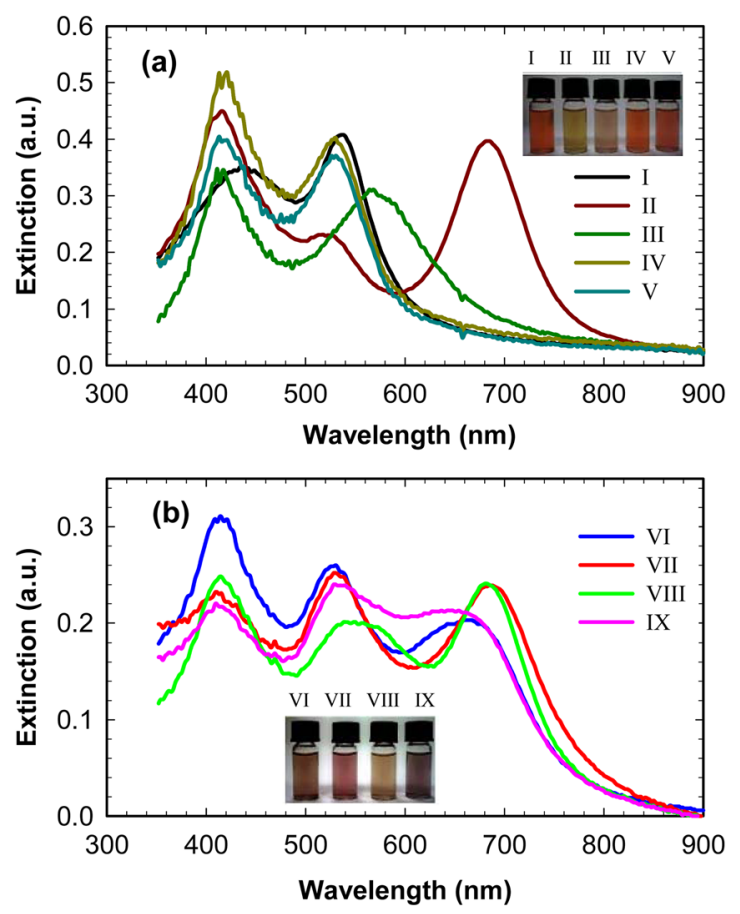

FIG. 3. (Color online) (a) and (b) Plasmonic response spectrum of multicomponent system (refer to Table II for composition details). ing from light trapping in photovoltaic devices to molecular detection in environmental and biological systems. Further, the PNGs could be also used to develop reconfigurable plasmonic devices using the optofluidics technology. ${ }^{15,16}$

This work was supported by the National Science Foundation through Grant CBET 1049454. We thank Dr. Hanbing Wang (Syracuse Biomaterials Institute) for her help in performing the rheological measurements and Dr. Kevin Yager (Brookhaven National Laboratory) for his valuable help in conducting the SAXS experiments. Syracuse University has filed a provisional U.S. patent application based on this study.

${ }^{1}$ H. A. Atwater and A. Polman, Nature Mater. 9, 205 (2010).

${ }^{2}$ S. Pillai et al., J. Appl. Phys. 101, 093105 (2007).

${ }^{3}$ J. Trice et al., Proc. SPIE 6648, 66480L (2007).

${ }^{4}$ E. M. Hicks et al., Nano Lett. 5, 1065 (2005).

${ }^{5}$ L. M. Campos et al., Adv. Mater. 20, 3728 (2008).

${ }^{6}$ Y. H. Lanyon et al., Anal. Chem. 79, 3048 (2007).

${ }^{7}$ Y. Wu et al., Langmuir 26, 11972 (2010).

${ }^{8}$ L. Kondic et al., Phys. Rev. E 79, 026302 (2009).

${ }^{9}$ C. Favazza et al., Appl. Phys. Lett. 91, 043105 (2007).

${ }^{10}$ J. Trice et al., Phys. Rev. Lett. 101, 17802 (2008).

${ }^{11}$ C. Favazza et al., Nanotechnology 17, 4229 (2006).

${ }^{12}$ A. I. Kuznetsov et al., Appl. Phys. Mater. Sci. Process. 94, 221 (2009).

${ }^{13}$ L. A. Porter et al., Nano Lett. 2, 1369 (2002).

${ }^{14} \mathrm{H}$. Krishna et al., MRS Proc. 960, 0960-N03-02.

${ }^{15}$ C. Monat et al., Nature Photon. 1, 106 (2007).

${ }^{16}$ D. Psaltis et al., Nature 442, 381 (2006).

${ }^{17}$ J. F. Berret, in Molecular Gels, edited by R. G. Weiss and P. Terech (Springer, the Netherlands, 2006), p. 667.

${ }^{18}$ M. E. Cates and S. J. Candau, J. Phys.: Condens. Matter 2, 6869 (1990).

${ }^{19}$ B. Nikoobakht and M. A. El-Sayed, Langmuir 17, 6368 (2001).

${ }^{20}$ F. Nettesheim et al., Langmuir 24, 7718 (2008).

${ }^{21}$ M. E. Helgeson et al., Langmuir 26, 8049 (2010).

${ }^{22}$ See supplementary material at http://dx.doi.org/10.1063/1.3615785 for discussion on SAXS and rheology experiments.

${ }^{23}$ A. V. Sangwai and R. Sureshkumar, Langmuir 27, 6638 (2011).

${ }^{24}$ P. Sahoo et al., Langmuir 25, 8742 (2009).

${ }^{25}$ P. Dastidar et al., Chem. Mater. 17, 741 (2005).

${ }^{26}$ S. H. Tung et al., Soft Matter 4, 1086 (2008).

${ }^{27}$ A. Einstein, Ann. Phys. 19, 289 (1906).

${ }^{28}$ C. F. Bohren and D. R. Huffman, Absorption and Scattering of Light by Small Particles (Wiley-VCH, Weinheim, Germany, 2004).

${ }^{29}$ J. M. Steele et al., "Plasmon hybridization in complex nanostructures," in Surface Plasmon Nanophotonics (Springer, Dordrecht, Netherlands, 2007), Chap. 13 